\title{
Evaluating student teaching style preferences in preparation for end of year exams: an analysis from Imperial College London
}

\author{
Chawla, J. \& Asumang, J. \\ Imperial College London, United Kingdom
}

\section{Background:}

Medical school teaching must strike a balance between adequately preparing students for both clinical practice and end-of-year assessments. Research suggests that traditional lecture-based teaching may not be the most efficient teaching method, in terms of knowledge retention and exam performance. Instead, intertwining gain of knowledge with application is beneficial. 'Single Best Answer' (SBA) teaching uses SBA-style questions to incorporate an interactive component to didactic teaching. We aimed to evaluate student preference on SBA and lecture-based teaching, assessing effectiveness in terms of subjective gain of knowledge and 'preparedness' for exams.

\section{Methods:}

Year 3 medical students at Imperial College London attended a peer-taught SBA-based tutorial series (September 2019 - March 2020). At the end of the series, feedback questionnaires focused on drawing comparisons between the style of teaching employed and the more traditional didactic lecture-based teaching delivered by the university. Thematic analysis of feedback was performed to determine student preference on teaching styles.

\section{Results:}

$81.8 \%(9 / 11)$ of students rated the series $5 / 5$ for usefulness and $90.9 \%(10 / 11)$ stated that they preferred SBA teaching to lecture-based teaching. Notably, $100 \%$ (11/11) felt better prepared for exams with an SBA-style of teaching. Qualitative analysis showed that students felt SBA teaching helped to better develop frameworks for approaching exam questions and allowed students to see how content could be translated into questions. Teaching was more engaging, interesting, and interactive whilst covering sufficient content. However, difficulties arose with this teaching style if there were weaknesses in the pre-existing knowledge base, with most students preferring an introductory lecture-based session prior to covering new topics.

\section{Conclusion:}

Evaluation of feedback suggested that the preferred method of learning may be a combination of 'introductory lecture-based teaching' and SBA-style application. Lectures provide sufficient theoretical understanding of new topics, while SBA teaching is subsequently preferred for developing exam technique.

Keywords: Assessment, SBA 